\title{
Pre-SN neutrino emissions from ONe cores in the progenitors of CCSNe
}

\author{
Chinami Kato ${ }^{1}$, S. Yamada ${ }^{1,2}$, H. Nagakura ${ }^{3}$, S. Furusawa ${ }^{4}$, \\ K. Takahashi ${ }^{5}$, H. Umeda ${ }^{5}$, T. Yoshida ${ }^{5}$ and K. Ishidoshiro ${ }^{6}$ \\ ${ }^{1}$ School of Advanced Science and Engineering, Waseda University, Japan \\ ${ }^{2}$ Advanced Research Institute for Science and Engineering, Waseda University, Japan \\ ${ }^{3}$ TAPIR, Walter Burke Institute for Theoretical Physics, California Institute of Technology, \\ USA \\ ${ }^{4}$ Frankfurt Institute for Advanced Studies, J.W. Goethe University, Germany \\ ${ }^{5}$ Department of Astronomy, The University of Tokyo, Japan \\ ${ }^{6}$ Research Center for Neutrino Science, Tohoku University, Japan
}

\begin{abstract}
In order to investigate the distinguishability about the progenitors of FeCCSNe and ECSNe, we calculate the luminosities and spectra of their pre-SN neutrinos and estimate the number of events at neutrino detectors.
\end{abstract}

Keywords. Supernovae:general, neutrinos

\section{Overview}

According to the stellar evolution theory, the progenitors with relatively light masses $\left(\lesssim 9 M_{\odot}\right)$ explode as ECSNe, while more massive ones explode as FeCCSNe. Then pre-SN neutrinos have important roles to distinguish two types of progenitors because neutrinos can escape freely from the core and deliver its information directly. Because of the recent development of detectional techniques, there is a possibility to detect them with low energy comparable to detectional threshold. Therefore, we focus on the distinguishability of SN-progenitors by the observation of pre-SN neutrinos.

We employ the realistic progenitor models until core bounce: a $9 M_{\odot}$ model for ECSN, 12 and $15 M_{\odot}$ models for FeCCSNe (Takahashi et al. (2013), Nagakura et al. (2014)). Based on their results, we calculate the luminosities and spectra of all-flavor neutrinos emitted via thermal and nuclear weak processes. Finally, we estimate the expected number at neutrino detectors including neutrino oscillation, assuming the distance to the progenitors to be $200 \mathrm{pc}$. It is demonstrated that $\bar{\nu}_{e}$ 's from the ECSNe-progenitors can hardly be detected at almost all detectors, whereas we will be able to detect $\sim 2500$ $\nu_{e}$ 's at DUNE. From the FeCCSN-progenitors, both $\nu_{e}$ 's and $\bar{\nu}_{e}$ 's will be detected: the number of $\bar{\nu}_{e}$ events will be largest for JUNO, 134-725 $\bar{\nu}_{e}$ 's, depending on the mass hierarchy whereas the number of $\nu_{e}$ events at DUNE is almost the same as that for the ECSN-progenitor. These results imply that the detection of $\bar{\nu}_{e}$ 's is useful to distinghish FeCCSN- from ECSN-progenitors, while $\nu_{e}$ 's will provide us with detailed information on core evolutions regardless of progenitor types (Kato et al. (2017)).

\section{References}

Kato, C., Yamada, S., Nagakura, H., Furusawa, S., Takahashi, K., Umeda, H., Yoshida, T., \& Ishidoshiro, K., 2017, in preparation

Takahashi, K., Yoshida, T., \& Umeda, H., 2013, ApJ, 771, 28

Nagakura, H., Sumiyoshi, K., \& Yamada, S., 2014, ApJS, 214, 16 